\title{
Experiments in haptic-based authentication of humans
}

\author{
Mauricio Orozco • Matthew Graydon • \\ Shervin Shirmohammadi • Abdulmotaleb El Saddik
}

Published online: 26 January 2008

(C) Springer Science + Business Media, LLC 2008

\section{Erratum to: Multimed Tools Appl \\ DOI 10.1007/s11042-007-0169-9}

The author photographs of Shervin Shirmohammadi and Abdulmotaleb El Saddik have been switched and appear with the incorrect biographies.

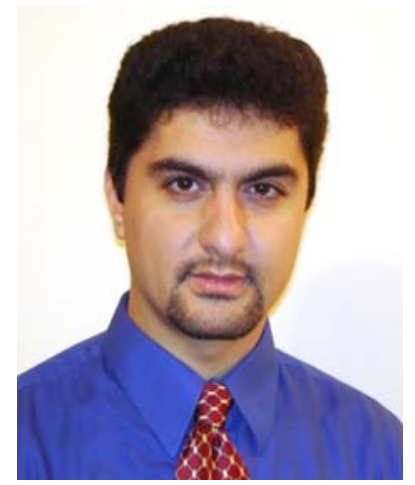

Shervin Shirmohammadi Ph.D., P.Eng., SMIEEE, received his Ph.D. in Electrical Engineering in 2000 from the School of Information Technology and Engineering, University of Ottawa, Canada, where he is currently an Assistant Professor. He has over 60 research publications, and is also an industry consultant with

The online version of the original article can be found at http://dx.doi.org/10.1007/s11042-007-0169-9.

M. Orozco $(\bowtie) \cdot$ M. Graydon $\cdot$ S. Shirmohammadi $\cdot$ A. El Saddik

Multimedia Communications Research Laboratory (MCRLab), University of Ottawa, Ottawa, Canada e-mail: morozco@mcrlab.uottawa.ca

S. Shirmohammadi

e-mail: shervin@mcrlab.uottawa.ca

A. El Saddik

e-mail: abed@mcrlab.uottawa.ca 
over a dozen technology transfers to the private sector. His current research interests include distributed multimedia computing and communications, P2P networking protocols, collaborative virtual presence and tele-haptics. he is an Associate Editor of the Journal of Multimedia Tools and Applications, Associate Editor of the Journal of Advanced Media and Communications, and Information Director of ACM Transactions on Multimedia Computing, Communications, and Applications (ACM TOMCCAP). He is a University of Ottawa Gold Medalist (highest convocation standing among all engineering students), a licensed Professional Engineer in Ontario, a Senior Member of the IEEE, and a Professional Member of the ACM.

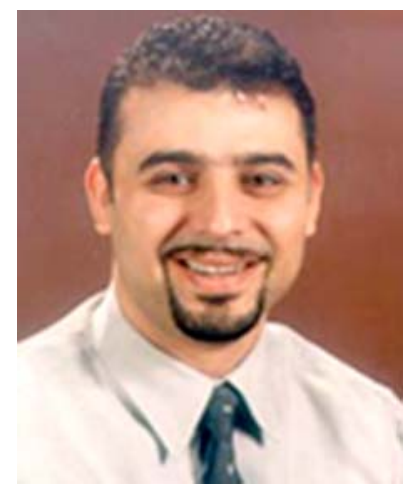

Abdulmotaleb El Saddik (IEEE M'02-SM'03) is an associate professor at the School of Information Technology and Engineering (SITE) at the University of Ottawa. He is the director of the Multimedia Communications Research Laboratory (MCRLab) and of the Information Technology Cluster, Ontario Research Network on Electronic Commerce. He is the founder of IEEE International workshop on Haptic Audio Visual Environments (HAVE) and a Distinguished IEEE Lecture. He is Editor of the International Journal of Advanced Media and Communication and Associate Editor of the ACM Journal of Educational Resources in Computing (JERIC). He serves in the program committee (as chair) of several ACM and IEEE conferences and workshops related to multimedia communications and Haptics. He is a recipient of the "Premier's Research Excellence Awards". 